\title{
Multi-Depots Vehicle Routing Problem with Simultaneous Delivery and Pickup and Inventory Restrictions: Formulation and Resolution
}

\author{
BOUANANE Khaoula $^{1}$, BENADADA Youssef ${ }^{2}$, BENCHEIKH Ghizlane $^{3}$ \\ Smart Systems Laboratory, Rabat IT Center, ENSIAS, Mohammed V University, Rabat, Morocco ${ }^{1,2}$ \\ Faculté des Sciences Juridiques Economiques et Sociales, Moulay Ismail University, Meknes, Morocco ${ }^{3}$
}

\begin{abstract}
Reverse logistics can be defined as a set of practices and processes for managing returns from the consumer to the manufacturer, simultaneously with direct flow management. In this context, we have chosen to study an important variant of the Vehicle Routing Problem (VRP) which is the Multi-Depot Vehicle Routing Problem with Simultaneous Delivery and Pickup and Inventory Restrictions (MD-VRPSDP-IR). This problem involves designing routes from multiple depots that simultaneously satisfy delivery and pickup requests from a set of customers, while taking into account depot stock levels. This study proposes a hybrid Genetic Algorithm which incorporates three different procedures, including a newly developed one called the K- Nearest Depot heuristic, to assign customers to depots and also the Sweep algorithm for routes construction, and the Farthest Insertion heuristic to improve solutions. Computational results show that our methods outperform the previous ones for MD-VRPSDP.
\end{abstract}

Keywords-Reverse logistic; inventory restrictions; VRPSDP; multi-depots version; Genetic Algorithm

\section{INTRODUCTION}

Our current production system is based on the use and processing of raw materials into finished products. The completion of this production cycle is through the final disposal or reuse of these products. This is how the last few years have seen the appearance of the emerging research problem: reverse logistics. Thus, issues related to efficiency and environmental effectiveness will have to be taken into consideration in the areas of business strategy, planning of the operation itself, as well as control of the distribution flows, in order to implement a reverse logistics of the product. Unlike the delivery of products to a customer, reverse logistics of returns is to manage flows from consumer to the manufacturer. The new challenges for researchers are to minimize transportation costs to make the reuse of products and materials more profitable than their elimination.

Reverse logistics can be defined as a set of practices designed to manage the return of products from customers to the manufacturer for repair, recycling or disposal at the lowest possible cost. To do this, a simple VRP is not adequate, it must be adapted to situations where vehicles can deliver end products and pick up returns simultaneously. The variant of the VRP most suited to this situation is the VRPSDP (Vehicle Routing Problem with Simultaneous Delivery and Pickup), where each customer is associated with delivery and pickup requests that must be made simultaneously.

In practice, applications of the VRPSDP are found especially within a reverse logistics context [1]. For instance, in the distribution system of food market chains [2], or in the urban public transport systems [3].

In this problem, each depot has a homogeneous vehicle fleet that must ensure the satisfaction of known delivery and pickup requests of a set of customers. Each customer must be visited once, this means that the vehicle upon arrived at the customer who must serve, the delivery and collection must be done at the same time. We assume that each depot is associated with a stock of products to be delivered and another for products collected from customers. The objective is to minimize the total distance traveled as well as the number of required vehicles while ensuring that the capacity constraints of vehicles and depots are not violated.

The MD-VRPSDP-IR is a very complex problem because it combines both the Multi-Depot version of the VRPSDP which is an NP hard problem and additional constraints such as inventory restrictions. To our knowledge, there is not yet a work in the literature that is interested in the interaction between these constraints: multiple depots, simultaneous delivery and pickup and inventory restrictions.

To avoid any confusion between certain variants of the VRP, we would like to clarify that the problem treated in this work is an extension of the VRPB (Vehicle Routing Problem with Backhauls), where the origin and the destination of all products delivered and picked up from customers are the depot. Unlike the VRPPD (Vehicle Routing Problem with Pickup and Delivery), where the interchanges of goods are made between customers.

In this paper, we propose a mathematical formulation as a Mixed Integer Linear Program (MILP), which aims to minimize both total travel cost and number of required vehicles. We implement the model in CPLEX to solve small problem instances optimally. Then, we propose a Hybrid Genetic Algorithm in which we use three different procedures to assign customers to depots, and then we embed the Sweep algorithm to construct routes for each depot and the Farther Insertion heuristic to improve the solution. The proposed heuristics are more complicated than those used for VRP 
involving only deliveries or pickups. The presence of combined delivery and pickup demands in our problem, and also restrictions on depot capacities mean that additional tests are required to preserve feasibility. The quality of our method is shown by tests on well-known benchmark instances of MDVRPSDP, which is special case of our problem and by comparison with optimal results, obtained by CPLEX as well as reported result for existing heuristics.

In Section 2, a rich literature review is detailed. In Section 3, mathematical formulation and notations of MDVRPSDP-IR are presented. Details of the proposed GAs are introduced in Section 4. In Section 5, the performance of the proposed GAs is examined by solving Gillett and Johnson's test problems and a computational example is represented with parameter settings. Section 6 concludes the paper with future works.

\section{RELATED LITERATURE REVIEW}

In this section, we propose to briefly discuss the literature of the VRPSDP and its Multi-Depot version, since we have not found a literature related to the MD-VRPSDP-IR.

VRPSDP is firstly introduced by [4]; he developed a model and a Cluster First - Route Second approach for the VRPSDP, and applied his model and the solution he proposed on a real case of a public library distribution system. Author in [1] discussed the importance of VRPSDP in the reciprocal logistic activities. He developed an Insertion-Based heuristic that use different criteria (travel distance, residual capacity and radial surcharge) to solve the problem. Afterward, many authors have become interested in the VRPSDP and its variants, and have developed several heuristics and metaheuristics to solve it. We mention here the most recent articles dealing with these problems. Author in [5] introduce the notion of Handling Cost in the VRPSDP; the items on the vehicle obeys the last-in-firstout policy, so handling operations are required if the delivery items are not the last loaded ones. They propose an Adaptive Large Neighborhood Search (ALNS) metaheuristic in which they embed the handling policies. Reference [6] deals with a special VRPSDP where three-dimensional loading constraints are assumed furthermore time windows constraints. To avoid any reloading effort, they consider two loading approaches of vehicles: loading from the backside with separation between delivery and pickup sections and loading at the long side. There method is a hybrid of an extended ALNS and conventional packing heuristics. Authors in [7] and [8] treat green VRPSDP; they propose models that minimize the cost of fuel consumption and pollutant emissions of vehicles. To solve his model, [7] uses Genetic Algorithms, which she hybrids with Sweep heuristic, and the Nearest Neighbor Heuristic to generate an initial population, and then Iterated Swap Procedure improves the chromosomes. Whereas, [8] applies the fuzzy approach when both pickup and delivery demands are uncertain, and they propose an ALNS heuristic. Reference [9] deals with a variant of the basic VRPSDP including the multiple trips and time windows characteristics. They propose a solution approach based on Tabu Search, with the sequential insertion algorithm to construct an initial solution. Other heuristics and metaheuristics have been proposed for different variants of VRPSDP; the most recent ones were published by [10]-[20].

Concerning the Multi-Depot version, we found in the literature that few studies. Starting with [21] who deal with the Multi-Depot case of simultaneous backhauling problems, their method consists of extending the classical Insertion-Based Heuristic to allow to the algorithm to insert more than one backhaul at a time. This method perform well for a small number of backhauls, but if this number increase, computational complexity increases rapidly. In [22], the author developed an integrated heuristic that treat linehaul and backhaul customers similarly.

Author in [23] proposed four Saving Based Algorithms for the Multi-Depot version of VRPSDP: Partition Based Algorithms, Nearest Customer Algorithm and two different Saving Based Algorithms. Author in [24] was the first to develop metaheuristics for the MDVRPSDP. The algorithm framework used in their procedure in based on the Iterated Local Search (ILS) with an Adaptive Neighborhood Selection mechanism (ANS). At first, they assign customers to their nearest depot for creating an initial solution, after, they apply Saving Algorithm to each depot. They used different structural neighborhood methods for improving and perturbation steps of ILS.

An Improved Genetic Algorithm (IGA) is developed in [25] to solve the MD-VRPSDP with Soft Time Windows. Firstly, customers are assigned to their nearest depot and initial solutions constructed by Scanning Algorithm. A greedy based strategy is used for cutting and merging routes. Finally, for optimizing and adjusting the feasible solutions, they used three neighborhood search methods and 3-opt local search.

To assign customers to depots, [26] employed the Minimum Cost Flow problem previously solved by a graph algorithm. In this way, the original problem becomes a set of several Single-Depot problems. After this, the Weber Basis Saving method is developed to construct the initial solution of each sub-problem. Finally, improvement phase is assured by the Modified Tabu Search.

At this point, we want to note that in the works cited above, concerning the Multi-Depot version of the VRPSDP, the authors assign customers to their nearest depots at first, then proceed to resolve each VRPSDP as a sub-problem. Our contribution in this paper is that we explore new ways to assign customers to depots while keeping a margin of randomness. More details are given in Section 4.

\section{PROBLEM DESCRIPTION AND FORMULATION}

The MD-VRPSDP-IR is the problem of construction routes for homogeneous vehicle fleets, which originate from several depots, visit a set of customers assigned to each depot, and return to the departure depot. The inventory restrictions constraint is reflected in the fact that each depot has two storage areas, one for the products that will be delivered to customers (SD: Stock for Deliveries), and the other for the products collected from customers (SP: Stock for Pickups). However, all goods transported must be taken from depots, and any collected returns must be sent to depots. The constraint assure that a customer can only be served if his delivery 
request is available in SD and his collection request has enough space to be stored in SP. Fig. 1 exemplifies the MD-VRPSDPIR with 2 depots and 14 customers. The brackets above the customers contain delivery and pickup demands, and those above the depots represent depot capacities of delivery and pickup demands.

Let $G(V, E)$ be a graph, where $V$ is the vertex set and $E=\{(i, j): i \neq j\}$ is the edge set. The vertex set $V$ is partitioned into two subsets $V_{d}=\{1, \ldots, m\}$ and $V_{c}=$ $\{m+1, \ldots, m+n\}$, which represent the set of depots and the set of customers, respectively. Each vertex $j \in V_{c}$ has a nonnegative pickup demand $\boldsymbol{P}_{\boldsymbol{j}}$, delivery demand $\boldsymbol{D}_{\boldsymbol{j}}$ and a service time $\boldsymbol{t}_{\boldsymbol{j}}$. Furthermore, in the depot vertex $j \in V_{d}$, there are no demands and service times $P_{j}=D_{j}=t_{j}=0$. For all $i, j \in V$, a distance matrix $\boldsymbol{d}_{\boldsymbol{i} \boldsymbol{j}}$ and a travel time matrix $\boldsymbol{t}_{\boldsymbol{i} \boldsymbol{j}}$ are associated with $E$. A set $K_{d}$ of identical vehicles of capacity $\boldsymbol{Q}$ is available at each depot $d \in V_{d}$. The optimal distribution of goods between depots and customers depends on inventory levels in depots, therefore each depot $d$ has maximum capacities $\boldsymbol{S} \boldsymbol{D}_{\boldsymbol{d}}$ and $\boldsymbol{S} \boldsymbol{P}_{\boldsymbol{d}}$ for delivery and pickup requests, respectively.

\section{A. Notions}

1) Sets

$\mathrm{V}_{\mathrm{d}}$ : Set of all depots.

$\mathrm{V}_{\mathrm{c}}$ : Set of all customers.

$\mathrm{V}:$ Set of all nodes, $\mathrm{V}=\mathrm{V}_{\mathrm{c}} \cup \mathrm{V}_{\mathrm{d}}$

$\mathrm{K}_{\mathrm{d}}$ : Set of vehicles associated with depot $\mathrm{d}$.

$\mathrm{K}$ : Set of all vehicles, $\mathrm{K}=\mathrm{U}_{\mathrm{d}} \mathrm{K}_{\mathrm{d}}$

\section{2) Indices}

D : Depot

$\mathrm{K}$ : Vehicle

I : Start node

$\mathrm{J}$ : Destination node

\section{3) Parameters}

$\mathrm{D}_{\mathrm{j}}$ : Delivery demand of customer $\boldsymbol{j}$

$\mathrm{P}_{\mathrm{j}}$ : Pick-up demand of customer $\boldsymbol{j}$

$t_{j}:$ Service time of customer $j$

$\mathrm{t}_{\mathrm{ij}}$ : Travel time of a vehicle from node $\boldsymbol{i}$ to node $\boldsymbol{j}$

$\mathrm{d}_{\mathrm{ij}}$ : Distance between node $\boldsymbol{i} \in \boldsymbol{V}$ and $\mathrm{j} \in \mathrm{V}$

$\mathrm{c}_{\mathrm{ij}}$ : Travel cost of a vehicle from node $\boldsymbol{i} \in \boldsymbol{V}$ to node $\mathrm{j} \in \mathrm{V}$

$C_{\text {Mil\$ }}$ : Mileage cost of a vehicle.

$\mathrm{C}_{\mathrm{k}}$ : Cost of operating vehicle $\boldsymbol{k}$.

Q: The maximum capacity of a vehicle.

$\mathrm{T}$ : The maximum working time allowed for a vehicle during a working day.

$\mathrm{SD}_{\mathrm{d}}$ : The maximum stock of delivery product in depot $\boldsymbol{d}$.

$\mathrm{SP}_{\mathrm{d}}$ : The maximum stock of picked up product in depot $\boldsymbol{d}$.

$\mathrm{M}:$ Large number.

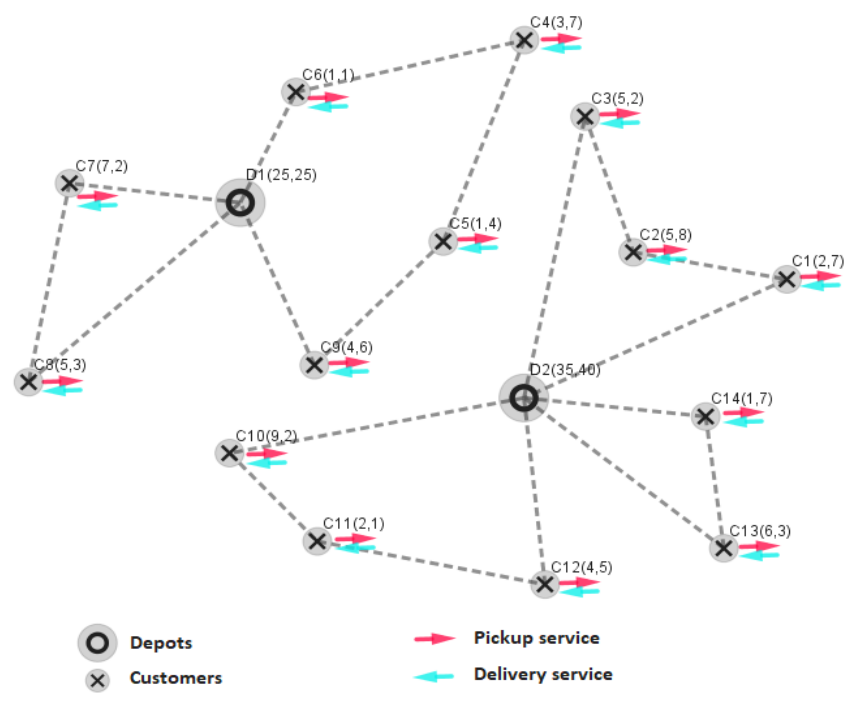

Fig. 1. Illustration of MD-VRPSDP-IR.

\section{4) Decision variables}

$\mathrm{x}_{\mathrm{ij}}^{\mathrm{k}}: \boldsymbol{x}_{\boldsymbol{i} \boldsymbol{j}}=\mathbf{1}$ when vehicle $\boldsymbol{k}$ travels directly from node $\boldsymbol{i} \in \boldsymbol{V}$ to node $\mathrm{j} \in \mathrm{V} . \boldsymbol{x}_{\boldsymbol{i} j}^{\boldsymbol{k}}=\mathbf{0}$ otherwise.

$L_{j}$ : Load of vehicle after having serviced customer $j \in V_{c}$.

$\mathrm{u}_{\mathrm{j}}$ : Variable used to prohibit sub tours; can be interpreted as position of node $\mathrm{j} \in V_{c}$ in the route.

$I L_{k}=\sum_{i \in V} \sum_{j \in V_{c}} D_{j} x_{i j}^{k} \quad(k \in K):$ Load of vehicle $\mathrm{k} \in \mathrm{K}$ when leaving the depot (Initial Load).

$F L_{k}=\sum_{i \in V_{c}} \sum_{j \in \mathrm{V}} P_{i} x_{i j}^{k} \quad(k \in K):$ Load of vehicle $\mathrm{k} \in \mathrm{K}$ after visiting last customer (Final Load).

\section{B. Mixed Integer Linear Programming Model for MD- VRPSDP-IR}

The objective of the proposed mathematical model is to minimize the total transportation cost $z$ due to the weighted sum of the total distance traveled of all vehicles and the cost related to the number of required vehicles, where $w_{d}$ and $w_{R}$ are the weight factors of the total distance traveled and the number of used vehicles, respectively, and $\alpha$ and $\beta$ are conversion factors from distance to cost (unit: $D \mathrm{~h} / \mathrm{km}$ ) and from number of vehicles to cost (unit: Dh/vehicle), respectively.

Minimize total $\operatorname{cost} z$ :

$$
z=w_{d} \cdot \alpha \sum_{k \in K} \sum_{i \in V} \sum_{j \in V} d_{i j} x_{i j}^{k}+w_{R} \cdot \beta \sum_{k \in K} \sum_{i \in V_{d}} \sum_{j \in V_{c}} x_{i j}^{k}
$$

Constraints of the problem are given below:

$$
\begin{array}{lr}
\sum_{k \in K} \sum_{i \in V} x_{i j}^{k}=1 & \left(j \in V_{c}\right) \\
\sum_{i \in V} x_{i s}^{k}=\sum_{j \in V} x_{s j}^{k} & \left(k \in K, s \in V_{c}\right) \\
\sum_{j \in V_{c}} x_{i j}^{k}=\sum_{l \in V_{c}} x_{l i}^{k} & \left(k \in K, i \in V_{d}\right) \\
\sum_{i \in V_{d}} \sum_{j \in V_{d}} x_{i j}^{k}=0 & (k \in K)
\end{array}
$$$$
\sum_{j \in V_{c}} t_{j} \sum_{i \in V} x_{i j}^{k}+\sum_{i \in V} \sum_{j \in V_{c}} t_{i j} x_{i j}^{k} \leq T \quad(k \in K)
$$ 
$u_{j} \geq u_{i}+1-(n+m)\left(1-\sum_{k \in K} x_{i j}^{k}\right) \quad\left(i, j \in V_{c}\right)$

Vehicle load constraints

$$
\begin{aligned}
& L_{j} \geq I L_{k}-D_{j}+P_{j}-M\left(1-x_{d j}^{k}\right) \quad\left(k \in K, d \in V_{d}, j \in V_{c}\right) \\
& L_{j} \geq L_{i}-D_{j}+P_{j}-M\left(1-\sum_{k \in K} x_{i j}^{k}\right) \quad\left(i, j \in V_{c}\right) \\
& I L_{k} \leq Q \quad(k \in K) \\
& L_{j} \leq Q \quad\left(j \in V_{c}\right)
\end{aligned}
$$

Inventory restrictions constraints

$$
\begin{array}{ll}
\sum_{k \in K_{d}} I L_{k} \leq S D_{d} & \left(d \in V_{d}\right) \\
\sum_{k \in K_{d}} F L_{k} \leq S P_{d} & \left(d \in V_{d}\right)
\end{array}
$$

Integrality constraints

$$
\begin{array}{ll}
x_{i j}^{k} \in\{0,1\} & (i, j \in V, k \in K) \\
u_{j} \geq 0 & \left(j \in V_{c}\right)
\end{array}
$$

Constraints (2) ensure that each customer is visited exactly once by exactly one vehicle. Flow conservation is ensured by constraint (3). Constraints (4) required that each vehicle starts and ends its route at the same depot. Constraints (5) impose that a vehicle cannot travel between two depots. Constraints (6) ensure that the total duration of each route (including travel time and service time) does not exceed a pre-set limit. Constraints (7) eliminate the sub-tours to ensure that the solution is connected. After visiting the first customer, the vehicle load is calculated by constraint (8) and after leaving other customers, the vehicle load is calculated by constraint (9). Constraints (10) and (11) ensure that the vehicle capacity is respected at each section of the route. Constraints (12) and (13) require that stock levels in each depot are not surpassed.

A necessary but not sufficient condition to have feasible solutions is to ensure that all customers can be served; this is verified by the following constraints:

$\sum_{j \in V_{c}} D_{j} \leq \sum_{d \in V_{d}} S D_{d}, \quad \sum_{j \in V_{c}} P_{j} \leq \sum_{d \in V_{d}} S P_{d}$

However, it is not worth adding them to the mathematical model, because we can deduce them from the constraints (2), (12) and (13).

\section{HYBRID GENETIC APPROACH}

The MD-VRPSDP-IR is a NP-hard problem. As the problem instances increase in size, the exact solution methods become highly time-consuming. In recent years, GA has been applied successfully to a wide variety of hard optimization problems such as the classical VRP and its multi-depot version. The success is mainly due to its simplicity, easy operations, and great flexibility. These are the major reasons why we selected a GA as an optimization tool in this paper.

The problem studied in this work is an integration of two hard optimization problems: grouping and routing problems. A simple GA may not perform well in this situation. Therefore, the GA developed in this paper is hybridized with several heuristics to construct and improve the solutions. Fig. 2 shows the flowchart of three Hybrid Genetic Algorithms (GAs). The difference between them is in the assignment of customers to depots: GA1 attribute customers randomly to depots, GA2 use the K-Nearest Depot heuristic to assign customers to depots considering the depot-customer distances, but also a random selection step and GA3 assign customers to their nearest depots.

\section{A. Chromosome Representation}

The permutation representation is used for genetic representation of the MD-VRPSDP-IR as shown in Fig. 3. A chromosome is built as an array with three rows: 1) customers, which are listed in the order in which they are visited; 2) depots, where customers are assigned depending on depot capacities; 3) vehicles required in each depot to satisfy all demands of customers assigned to this depot. Routes are determined depending on vehicles capacity. The number of customer nodes determines the length of the chromosome.

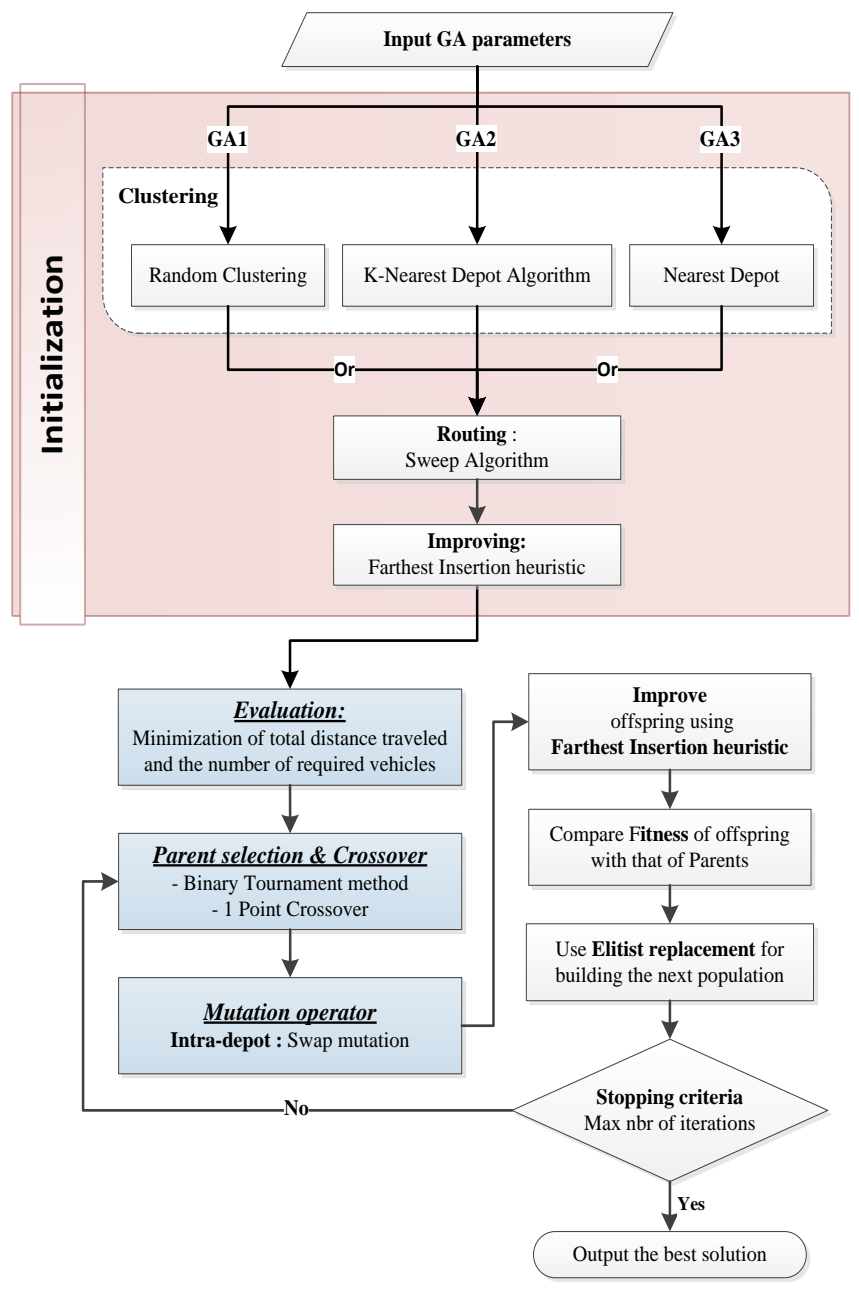

Fig. 2. The Flowchart of Gas.

\section{B. Initial Population Construction}

In this work, there are three phases to generate a feasible initial solution (Fig. 4). The first one is to assign customers to depots, that is, the grouping problem, for this, we use one of the three procedures mentioned above. The second phase is to perform, for each depot, a clustering of customers assigned to 
this depot and then determine a vehicle route for each cluster by using the Sweep algorithm, that is, the routing problem. The last phase consists of improvement of several routes already built, for this we use the Farthest Insertion Heuristic.

1) Grouping: It is worth to note that the grouping problem and the routing problem in the "cluster first, route second" approach are not independent. A bad assignment solution will result in routes of higher total cost (distance) than with a better assignment. The grouping procedures described in the following assign customers to depots so that the capacity of the depots is not exceeded.

Grouping can be done using one of the following three methods: 1) Attributing customers randomly to depots: we randomly choose a customer and then a depot, if the depot capacity is not yet reached, we assign this customer to this deposit, otherwise we choose another deposit and so on. 2) Using the K-Nearest Depot heuristic (See next paragraph). 3) Assigning customers to their nearest depots within the limit of stock availability in each depot.

2) The k-Nearest depot heuristic: We developed this algorithm to assign all customer to different depots based on the customer-depot distance, while keeping a random side in the procedure, as shown in Fig. 5. For each customer, we find the $\frac{m}{2}$ (where $m$ is the number of depots) closest depots of this customer and who can serve it obviously. Then we randomly choose one of these depots to assign the customer. We first check the feasibility of this assignment, if the capacities of the depot allow this assignment, it is done, if not, we choose another deposit, and so on.

3) Routing: the sweep algorithm: The sweep algorithm belongs to the Cluster First - Route Second family. It begins by assigning to customers angular coordinates related to depot, and then scanning in the direction of increasing coordinates. In our paper, to order customers, we do not assign them polar coordinates, we use the order generated in the grouping phase.

Customers are added successively to a vehicle route following this order, and as soon as the capacity of the vehicle is reached, a new vehicle route is created and the process is repeated until all customers have been swept. Then, when all routes are formed, we execute the next phase.

4) Improving: the farthest insertion heuristic: After the construction is finished, routing costs can be reduced using a route improvement algorithm. In our improvement method, before validating a change, we must verify that the capacity of the vehicles performing the tours processed is respected in all points and that the change brings a gain in the cost of the solution.

In the FI heuristic, a route is constructed by progressively adding a customer one at a time until a complete route is formed. The part of the route that is already built remained unchanged during the tour construction process. The FI heuristic start with a route of two customers those are located farthest to one another. Then, an unvisited customer that is farthest to the route is selected. This customer is inserted between two consecutives customers that result in minimum increase of route cost.

\begin{tabular}{|c|c|c|c|c|c|c|c|c|c|c|c|c|c|c|}
\hline Customers & 5 & 2 & 10 & 14 & 9 & 11 & 12 & 3 & 6 & 7 & 4 & 8 & 13 & 1 \\
\hline Depots & 1 & 2 & 2 & 2 & 1 & 2 & 2 & 2 & 1 & 1 & 1 & 1 & 2 & 2 \\
\hline Vehicles & 2 & 3 & 5 & 4 & 2 & 5 & 5 & 3 & 2 & 1 & 2 & 1 & 4 & 3 \\
\hline
\end{tabular}

Fig. 3. Chromosome Representation.

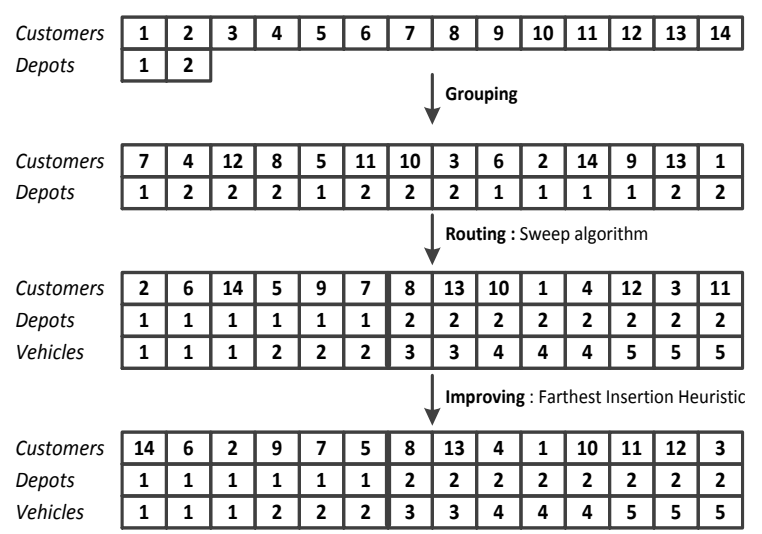

Fig. 4. Initialization of Gas.

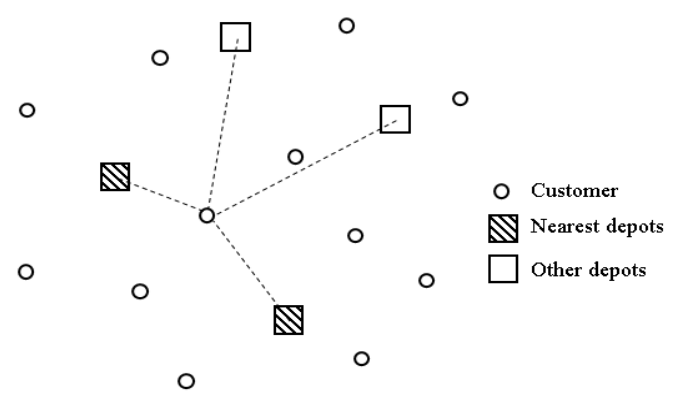

Fig. 5. The K-Nearest Depots Heuristic.

\section{Fitness Function}

Fitness function represents the method for the evaluation of individuals. Since each generated chromosome is a feasible solution, and our function combines route length with other parameter, that is the number of required vehicles, the fitness value of each chromosome is then calculated with weighted sum of all parameters [27]. This method requires adding the values of fitness functions together using weighted coefficients for each individual objective. That is, our multi-objective MDVRPSDP-IR is transformed into a single-objective optimization problem, where the fitness function $F(x)$ of an individual $x$ is returned as:

$F(x)=\left(1+\left(w_{d} \cdot \alpha \cdot \sum_{k \in R} D_{k}+w_{R} \cdot \beta \cdot|R|\right)\right)^{-1}$

where $D_{k}=\sum_{i \in V} \sum_{j \in V} d_{i j} x_{i j}^{k}$

$w_{d}$ and $w_{R}$ are weight parameters associated with the total traveled cost of all vehicles and the number of required vehicles, respectively. The weight values of the parameters used in this function were established empirically. 


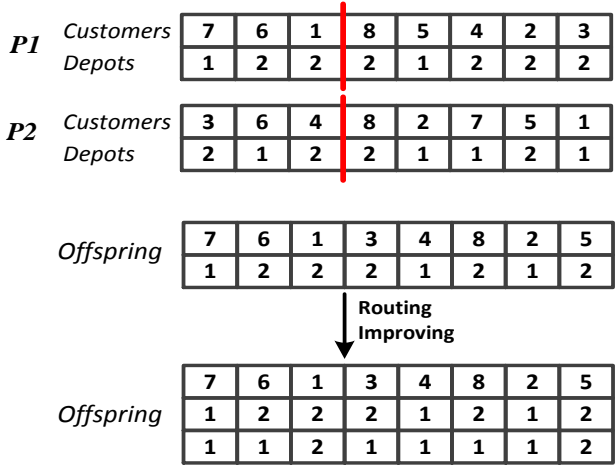

Fig. 6. Example of the $1 \mathrm{X}$ Crossover.

\section{Parent Selection and Crossover}

Parent selection is performed through a binary tournament, which twice randomly chooses two individuals from the population, and keeps the one with the highest value of fitness. This process is repeated until the required number of individuals is obtained.

In this paper, we use the One Point Crossover (1X). The crossing operator is applied just on the first two range of the chromosome; those of customers and depots, as shown in Fig. 6. Afterwards, routing and improving procedures are applied to the offspring to build the routes for each depot. To build the offspring, we first start with the row of customers; the first part of the first parent is copied, and then the elements of the second part of this parent are reordered in the order of appearance they have in the second parent. Afterwards, the allocation of customers to depots is done by respecting the depots capacities; for each client, we first check if it can be assigned to its initial depot (in the first parent), otherwise we choose another nearest depot, and so on.

\section{E. Mutation}

The mutation operator plays the role of a disruptive element; it explores a wider search space and allows maintaining the diversity of the next population, avoiding the algorithm to converge too quickly towards a local optimum. We employed the Swap mutation, which we applied as an intra-depot mutation that involves a single depot. Swap mutation is simple; it consists of randomly taking two genes (2 customers) from the chromosome and swapping them. If the offspring is not feasible, it is deleted.

\section{COMPUTATIONAL Results AND Discussions}

This section describes computational experiments carried out to study the performance of the proposed GAs. The algorithm is coded in $\mathrm{C}$ and run on a laptop computer with an Intel Core i7 $2.9 \mathrm{GHz}$ processor with $8 \mathrm{~GB}$ RAM, under the operating system Windows ${ }^{\circledR} 7$. First, we compare the performance of GAs, which have the best results will be used in the tests that follow. Then, to validate the MILP model for the MD-VRPSDP-IR proposed in this paper, we compare our GA results with those obtained by CPLEX, for a small instance, through an illustrative example. To assess the effectiveness of the best GA, it is tested on its special case MD-VRPSDP, since we did not find reported results for MD-
VRPSDP-IR. For this, we assume that depot capacities are infinite. And then we compare results obtained by the best GA with [21] and [23] for which there are reported results for the MD-VRPSDP, and are using the same data.

\section{A. Benchmarks}

For the numerical experiments, we adopt the data set provided by [21] as the tested instances. It includes 22 problem instances ( 2 to 5 depots, 50 to 249 customers) generated from 11 benchmark problems of [28] (the first 8 ones are provided from [29] and the last 4 ones from [30]). The 22 problem instances are partitioned as sets $\mathrm{X}$ and $\mathrm{Y}$ based on the difference of deliveries and pickups.

We use the method proposed by [21] and used by [23] for splitting the original demand into pickup and delivery demands. Let $x_{i}$ and $y_{i}$ denote the coordinates of customeri, and let $D_{i}^{\text {org }}$ denote the demand for customer $i$ in the original problem. The distance matrix is generated using the original coordinates and is calculated with Euclidean distance. However, $D_{i}^{\text {org }}$ is split into delivery demand $D_{i}$ and pickup demand $P_{i}$ as follows:

$D_{i}=r_{i} \times D_{i}^{\text {org }}$ and $P_{i}=\left(1-r_{i}\right) \times D_{i}^{\text {org }}$

where $r_{i}=\min \left(\frac{x_{i}}{y_{i}}, \frac{y_{i}}{x_{i}}\right)$

In this way, set $X$ of 11 instances is generated. The other set $\mathrm{Y}$, likewise with 11 instances, is generated by exchanging the pickup and delivery demands in problem instances of set $X$. The basic characteristics of instances are shown in Table 1.

In addition to these characteristics, we will need the storage capacity SD of products to be delivered and the storage capacity SP of the collected products, for each depot and each instance. The SD and SP values used are created by ourselves and are compatible with the instance characteristics and the conditions of the problem. We assume that the values of SD and SP are equal for all the depots of the same instance. Depots' information is as Table 2 shows.

TABLE I. BASIC CHARACTERISTICS OF DATA SETS FOR THE MDVRPSDP

\begin{tabular}{|l|l|l|l|l|}
\hline N $^{\circ}$ Inst. & n & d & Q & Depot coordinates \\
\hline GJ1 & 50 & 4 & 80 & $(20,20),(30,40),(50,30),(60,50)$ \\
\hline GJ2 & 50 & 4 & 160 & $(20,20),(30,40),(50,30),(60,50)$ \\
\hline GJ3 & 75 & 5 & 140 & $(40,40),(50,22),(55,55),(25,45),(20,20)$ \\
\hline GJ4 & 100 & 2 & 100 & $(35,20),(35,50)$ \\
\hline GJ5 & 100 & 2 & 200 & $(15,35),(55,35)$ \\
\hline GJ6 & 100 & 3 & 100 & $(15,20),(50,20),(35,55)$ \\
\hline GJ7 & 100 & 4 & 100 & $(15,35),(55,35),(35,20),(35,50)$ \\
\hline GJ8 & 249 & 2 & 500 & $(-33,33),(33,-33)$ \\
\hline GJ9 & 249 & 3 & 500 & $(70,0),(-50,60),(-50,-60)$ \\
\hline GJ10 & 249 & 4 & 500 & $(75,0),(0,75),(-75,0),(0,-75)$ \\
\hline GJ11 & 249 & 5 & 500 & $(70,0),(40,-80),(40,80),(-60,20),(-60,-20)$ \\
\hline
\end{tabular}


TABLE II. DEPOT'S INFORMATION

\begin{tabular}{|l|l|l||l|l|l|}
\hline $\mathbf{N}^{\circ}$ Inst. & SD & SP & N $^{\circ}$ Inst. & SD & SP \\
\hline GJ1X & 120 & 85 & GJ1Y & 85 & 120 \\
\hline GJ2X & 120 & 85 & GJ2Y & 85 & 120 \\
\hline GJ3X & 170 & 120 & GJ3Y & 120 & 170 \\
\hline GJ4X & 440 & 320 & GJ4Y & 320 & 440 \\
\hline GJ5X & 440 & 320 & GJ5Y & 320 & 440 \\
\hline GJ6X & 290 & 215 & GJ6Y & 215 & 290 \\
\hline GJ7X & 215 & 160 & GJ7Y & 160 & 215 \\
\hline GJ8X & 3050 & 3100 & GJ8Y & 3100 & 3050 \\
\hline GJ9X & 2040 & 2070 & GJ9Y & 2070 & 2040 \\
\hline GJ10X & 1530 & 1550 & GJ10Y & 1550 & 1530 \\
\hline GJ11X & 1220 & 1240 & GJ11Y & 1240 & 1220 \\
\hline
\end{tabular}

\section{B. Parameter Settings}

First, we employ GJ1X instance to determine the appropriate number of iterations (Nbr_Iter) and population size (Pop_Size) for GAs, we test combinations:

Pop $p_{\text {Size }}=\{50,100,150,200\}$

Nbr_Iter $=\{300,500,1000,5000\}$

Results of several iterations are summarized in Table 3. For each combination, we run the program 30 times, the best objective function value and the average of all objective function values are summarized in column I and II, respectively. The computation time is given as average CPU times (s).

From these results, considering objective function values, the best solutions are given by the combination 500-5000 (Pop_Size-Nbr_Iter) as well as by the combination 200-300. However, combination 200-300 is preferable when considering also CPU time; it has a much less important CPU time than the combination 500-5000. Therefore, we use the combination 200-300 for Instances GJ1 to GJ7 and the combination 5005000 for instances GJ8 to GJ11.

The other parameters used in GAs are crossover rate $p_{c}=0.7$ and mutation rate $p_{m}=0.01$. To obtain these values, we proceeded in the same way as for the population size and the number of iterations; we test combinations of $p_{c}=$ $\{0.5,0.6,0.7,0.8\}$ and $p_{m}=\{0.01,0.05,0.1\}$, the same instance GJ1X is employed to test them by changing the value of one parameter while keeping the other fixed. These values are then used in all other tests.

\section{Experiments and Results}

1) Comparison of GAs performances: A computational study is carried out to compare GA1 with random assignment of customers to depots, GA2 using the K-ND heuristic and GA3 which assign customers to the nearest depot. Table 4 reports the best solutions for the MD-VRPSPD-IR. To obtain the routing cost (Routing \$) without taking into account the cost of using vehicles, we set the conversion factors at $\alpha=1$ and $\beta=0$. After, we calculate the total transportation cost (Trans \$) considering the number of used vehicles using the conversion factors $\alpha=1$ and $\beta=100$, as follows:

\section{Trans $\$=(\alpha *$ Routing $\$)+(\beta * N b r$ of Vehicles $)$}

By comparing the routing costs, we find that the results given by GA1 are very high, and therefore are not competitive with those of GA2 and GA3. As for CPU time, it undergoes an insignificant change. GA2 gives better results than GA3 (in most cases). GA2 is also preferable when considering the number of required vehicles; it is usually smaller for GA2 than for GA3. We opted for a weighted sum of the routing cost and the number of used vehicles to compare the performance of GAs. It is found that the performance of GA2 is superior to that of GA3 in terms of total cost of transportation within nearly equal average computational time. The best solutions generated by GA2 are much better than those generated by GA3, this is due to the fact that GA2 incorporates the K-ND heuristic, which affects customers to depots taking into account the depot-customer distances, but also leaves a side of random. If we assign customers to the nearest depot, the assignments will always be the same for a given instance, and this will decrease the performance of the algorithm because it prevents it from exploring more, and thus excludes much solutions.

TABLE III. COMPUTATIONAL RESULTS FOR COMBINATIONS OF POPULATION SIZE AND NUMBER OF ITERATIONS.

\begin{tabular}{|l|l|l|l|l|}
\hline Pop_Size & Nbr_Iter & I & II & CPU \\
\hline 50 & 300 & 382 & 419 & 0,12 \\
\hline 100 & 300 & 386 & 407 & 0,13 \\
\hline 150 & 300 & 377 & 399 & 0,13 \\
\hline 200 & 300 & $\mathbf{3 5 5}$ & $\mathbf{3 8 8}$ & $\mathbf{0 , 1 6}$ \\
\hline 50 & 500 & 396 & 414 & 0,17 \\
\hline 100 & 500 & 373 & 410 & 0,18 \\
\hline 150 & 500 & 383 & 400 & 0,16 \\
\hline 200 & 500 & 382 & 398 & 0,20 \\
\hline 50 & 1000 & 393 & 410 & 0,23 \\
\hline 100 & 1000 & 389 & 408 & 0,24 \\
\hline 150 & 1000 & 370 & 395 & 0,26 \\
\hline 200 & 1000 & 364 & 397 & 0,31 \\
\hline 50 & 5000 & 391 & 418 & 0,75 \\
\hline 100 & 5000 & 376 & 401 & 0,86 \\
\hline 150 & 5000 & 371 & 393 & 0,98 \\
\hline 200 & 5000 & $\mathbf{3 5 2}$ & $\mathbf{3 8 3}$ & $\mathbf{1 , 5 2}$ \\
\hline & & & & \\
\hline
\end{tabular}


TABLE IV. COMPARISON OF GAS PERFORMANCES FOR MD-VRPSDP-IR

\begin{tabular}{|c|c|c|c|c|c|c|c|c|c|c|c|c|}
\hline \multirow[b]{2}{*}{ Instance } & \multicolumn{4}{|l|}{ GA1 } & \multicolumn{4}{|l|}{ GA2 } & \multicolumn{4}{|l|}{ GA3 } \\
\hline & Total \$ & Routing \$ & Nbr_Veh & $\mathrm{CPU}$ & Total \$ & Routing \$ & Nbr_Veh & $\mathrm{CPU}$ & Total \$ & Routing \$ & Nbr_Veh & $\mathrm{CPU}$ \\
\hline GJ1X & 1309 & 509 & 8 & 0,35 & 1079 & 279 & 8 & 0,35 & 1189 & 389 & 8 & 0,45 \\
\hline GJ2X & 721 & 321 & 4 & 0,37 & 565 & 165 & 4 & 0,28 & 605 & 205 & 4 & 0,41 \\
\hline GJ4X & 2839 & 1639 & 12 & 0,44 & 2494 & 1294 & 12 & 0,36 & 2520 & 1320 & 12 & 0,41 \\
\hline GJ5X & 1539 & 939 & 6 & 0,53 & 1308 & 708 & 6 & 0,42 & 1334 & 734 & 6 & 0,42 \\
\hline GJ8X & 6487 & 4587 & 19 & 3,04 & 5417 & 3517 & 19 & 3,15 & 5374 & 3474 & 19 & 3,19 \\
\hline GJ9X & 6327 & 4427 & 19 & 3,06 & 4895 & 3195 & 17 & 2,85 & 5295 & 3395 & 19 & 3,06 \\
\hline GJ10X & 6258 & 4358 & 19 & 3,01 & 4905 & 3005 & 19 & 3,28 & 5282 & 3382 & 19 & 3,17 \\
\hline GJ11X & 6059 & 4159 & 19 & 2,93 & 4707 & 2907 & 18 & 3,11 & 5199 & 3299 & 19 & 3,38 \\
\hline Average & 3460 & 2205 & 12,5 & 1,36 & 2759 & 1540 & 12,2 & 1,36 & 2956 & 1692 & 12,6 & 1,43 \\
\hline GJ5Y & 1587 & 987 & 6 & 0,43 & 1302 & 702 & 6 & 0,33 & 1341 & 741 & 6 & 0,42 \\
\hline GJ6Y & 2695 & 1495 & 12 & 0,39 & 1935 & 835 & 11 & 0,41 & 2217 & 1017 & 12 & 0,46 \\
\hline GJ7Y & 2472 & 1272 & 12 & 0,41 & 1993 & 793 & 12 & 0,42 & 2176 & 976 & 12 & 0,63 \\
\hline GJ8Y & 6451 & 4551 & 19 & 2,91 & 5389 & 3489 & 19 & 2,89 & 5388 & 3488 & 19 & 3,37 \\
\hline GJ9Y & 6389 & 4489 & 19 & 2,92 & 4878 & 3178 & 17 & 2,97 & 5355 & 3355 & 20 & 3,43 \\
\hline GJ10Y & 6312 & 4412 & 19 & 3,04 & 4860 & 2960 & 19 & 3,24 & 5287 & 3387 & 19 & 2,96 \\
\hline GJ11Y & 6204 & 4304 & 19 & 2,97 & 4711 & 2911 & 18 & 3,19 & 5186 & 3286 & 19 & 3,42 \\
\hline Average & 3491 & 2237 & 12,5 & 1,32 & 2748 & 1529 & 12,2 & 1,36 & 2960 & 1697 & 12,6 & 1,47 \\
\hline
\end{tabular}

It is very important to note that the value assigned to the conversion factor $\beta$ is set arbitrarily to 100 (a small value) just to show that the number of required vehicles in each solution is as important as the routing cost, and may even be larger when the value of $\beta$ increases, which is the case in reality. That said, when the value of $\beta$ increases, it directly and significantly affects the total cost of transportation. You can easily notice that if we increase the value of the conversion factor $\beta$, the results will switch quickly to a much higher performance for GA2 than for GA3, because in most instances, GA2 uses fewer vehicles than GA3, which proves the efficiency and strength of the developed K-ND heuristic.

2) Comparison with CPLEX: We use an illustrative example, with 2 depots and 12 customers, to compare the results obtained by CPLEX with those of GA2. Location of depots and customers and delivery and pick-up demands of customers are shown in Figs. 7 and 8, respectively.

Vehicle capacity is set at 80 and depot capacities are set at SD_1=SD_2=100 and SP_1=SP_2=50. To obtain the routing cost, conversion factors are set at $\alpha=1$ and $\beta=0$. Results are summarized in Table V and illustrated in Fig. 9. Four vehicles served 12 customers, 2 for each depot.

We can easily notice that the results obtained by the algorithm developed in this paper are very close to the optimal value obtained by CPLEX solver, which uses branch and bound algorithm for solving MILP models. In addition, the proposed algorithm gives better solutions within significantly shorter time frame.

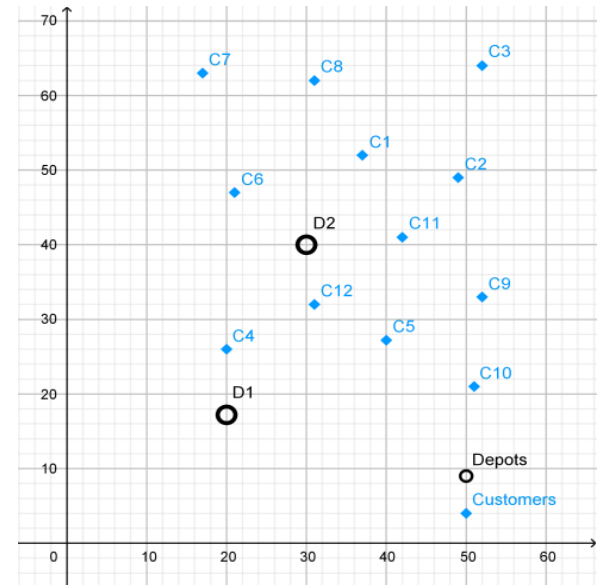

Fig. 7. Locations of Depots and Customers.

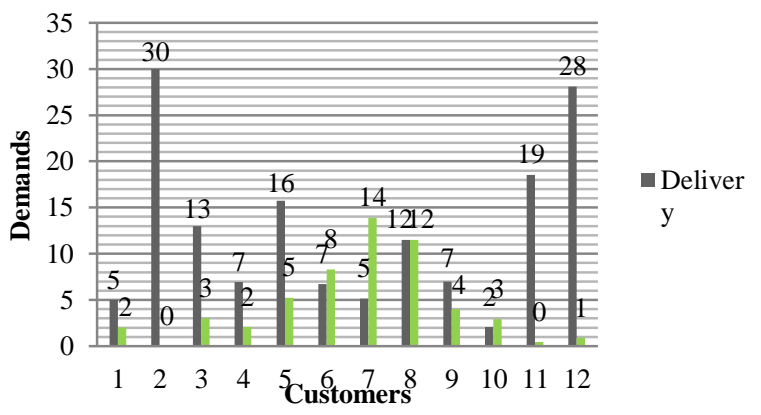

Fig. 8. Delivery and Pickup Demands of Customers. 

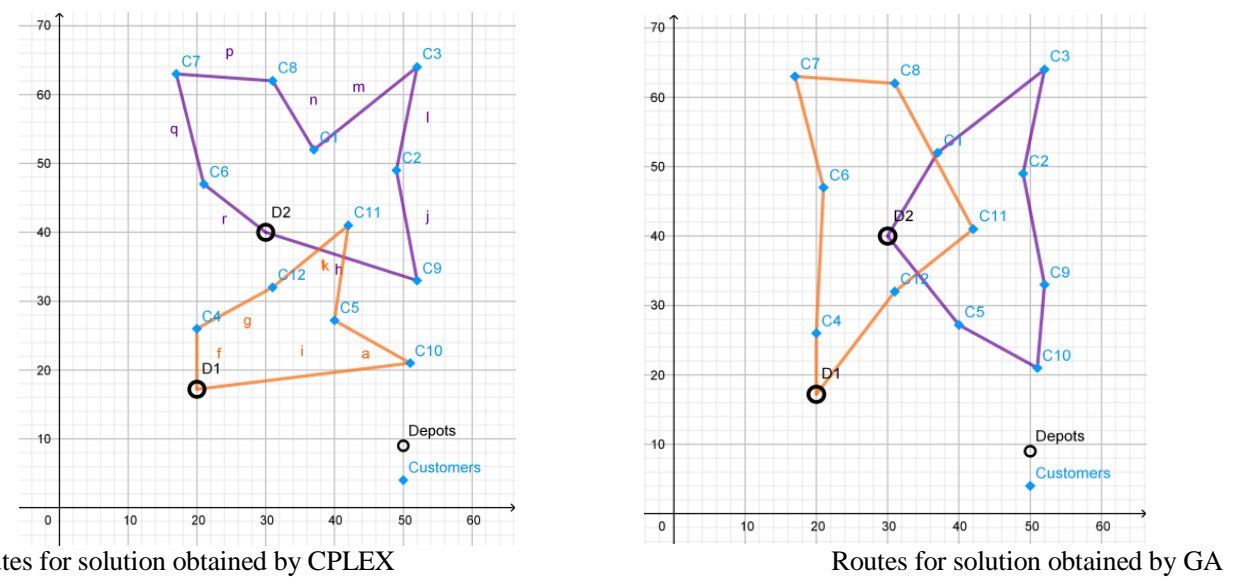

Fig. 9. Illustration of Results for Instance with 2 Depots and 12 Customers.

TABLE V. COMPARISON OF RESULTS OF CPLEX AND GA

\begin{tabular}{|l|l|l|l|}
\hline & Routes & Routing \$ & CPU \\
\hline \multirow{2}{*}{ CPLEX } & $\{\mathrm{D} 1-\mathrm{C} 4-\mathrm{C} 12-\mathrm{C} 11-\mathrm{C} 5-\mathrm{C} 10-\mathrm{D} 1\}$ & \multirow{2}{*}{221} & 2 \\
\cline { 2 - 4 } & $\{\mathrm{D} 2-\mathrm{C} 9-\mathrm{C} 2-\mathrm{C} 3-\mathrm{C} 1-\mathrm{C} 8-\mathrm{C} 7-\mathrm{C} 6-\mathrm{D} 2\}$ & & \\
\hline \multirow{2}{*}{ GA2 } & $\{\mathrm{D} 1-\mathrm{C} 4-\mathrm{C} 6-\mathrm{C} 7-\mathrm{C} 8-\mathrm{C} 11-\mathrm{C} 12-\mathrm{D} 1\}$ & 222,3 & \multirow{2}{*}{$0,09 \mathrm{~s}$} \\
\cline { 2 - 5 } & $\{\mathrm{D} 2-\mathrm{C} 1-\mathrm{C} 3-\mathrm{C} 2-\mathrm{C} 9-\mathrm{C} 10-\mathrm{C} 5-\mathrm{D} 2\}$ & & \\
\hline
\end{tabular}

3) Computational results and performance analysis: The objective is to minimize the weighted sum of the travel distances and the number of required vehicles. We assume that depot capacities are infinite. To calculate the total transportation cost, we set conversion factors $\alpha$ equal to 1 and $\beta$ equal to 100 . Results are reported in Table 6.

Unlike the results obtained for the MD-VRPSDP-IR, and by comparing the routing costs, we find that GA3 gives better results than GA2 (in most cases). However, GA2 is preferable when considering the number of required vehicles. Consequently, the performance of GA2 remains higher to that of GA3 in terms of total cost of transportation, even though its routing cost is slightly worse than that of GA3.

TABLE VI. GA2 AND GA3 PERFORMANCES FOR MD-VRPSDP

\begin{tabular}{|c|c|c|c|c|c|c|c|c|}
\hline \multirow[b]{2}{*}{ Instance } & \multicolumn{4}{|l|}{ GA2 } & \multicolumn{4}{|l|}{ GA3 } \\
\hline & Total \$ & Routing \$ & Nbr_Veh & CPU & Total \$ & Routing \$ & Nbr_Veh & $\mathrm{CPU}$ \\
\hline GJ1X & 1206 & 406 & 8 & 0,36 & 1273 & 373 & 9 & 0,39 \\
\hline GJ2X & 531 & 131 & 4 & 0,36 & 625 & 125 & 5 & 0,31 \\
\hline GJ3X & 1254 & 454 & 8 & 0,38 & 1446 & 446 & 10 & 0,41 \\
\hline GJ4X & 2054 & 854 & 12 & 0,41 & 2102 & 902 & 12 & 0,47 \\
\hline GJ5X & 1315 & 715 & 6 & 0,42 & 1427 & 727 & 7 & 0,36 \\
\hline GJ6X & 2398 & 1098 & 13 & 0,39 & 2475 & 1175 & 13 & 0,42 \\
\hline GJ7X & 2092 & 892 & 12 & 0,43 & 2331 & 1031 & 13 & 0,43 \\
\hline GJ8X & 5406 & 3506 & 19 & 2,76 & 5451 & 3451 & 20 & 2,82 \\
\hline GJ9X & 5332 & 3332 & 20 & 2,79 & 5216 & 3216 & 20 & 2,87 \\
\hline GJ10X & 4778 & 2878 & 19 & 2,77 & 4861 & 2861 & 20 & 3,2 \\
\hline GJ11X & 4221 & 2321 & 19 & 2,85 & 4272 & 2272 & 20 & 3,82 \\
\hline Average & 2781 & 1508 & 12,7 & 1,27 & 2862 & 1507 & 13,5 & 1,41 \\
\hline GJ1Y & 1052 & 352 & 7 & 0,37 & 1309 & 409 & 9 & 0,36 \\
\hline GJ2Y & 539 & 139 & 4 & 0,34 & 629 & 129 & 5 & 0,37 \\
\hline GJ3Y & 1219 & 419 & 8 & 0,45 & 1457 & 457 & 10 & 0,37 \\
\hline GJ4Y & 2032 & 832 & 12 & 0,38 & 2325 & 1125 & 12 & 0,42 \\
\hline GJ5Y & 1338 & 738 & 6 & 0,42 & 1415 & 715 & 7 & 0,38 \\
\hline GJ6Y & 2197 & 997 & 12 & 0,38 & 2251 & 951 & 13 & 0,38 \\
\hline GJ7Y & 2025 & 825 & 12 & 0,45 & 2319 & 1019 & 13 & 0,39 \\
\hline GJ8Y & 5417 & 3517 & 19 & 2,82 & 5471 & 3471 & 20 & 2,87 \\
\hline GJ9Y & 5395 & 3395 & 20 & 2,95 & 5316 & 3316 & 20 & 2,73 \\
\hline GJ10Y & 4883 & 2983 & 19 & 2,92 & 4881 & 2881 & 20 & 3,95 \\
\hline GJ11Y & 4377 & 2477 & 19 & 3,59 & 4412 & 2412 & 20 & 3,05 \\
\hline Average & 2770 & 1516 & 12,5 & 1,37 & 2890 & 1535 & 13,5 & 1,39 \\
\hline
\end{tabular}


TABLE VII. COMPARISON OF THE AVERAGE RESULTS FOR THE MD-VRPSDP

\begin{tabular}{|c|c|c|c|c|c|c|c|c|c|c|c|c|}
\hline \multirow{2}{*}{ Instances } & \multicolumn{4}{|c|}{ Salhi and Nagy (1999) } & \multicolumn{4}{|c|}{ Gajpal and Abad (2009) } & \multicolumn{4}{|l|}{ GA2 } \\
\hline & Total \$ & Routing \$ & Nbr_Veh & $\mathrm{CPU}$ & Total \$ & Routing \$ & Nbr_Veh & $\mathrm{CPU}$ & Total \$ & Routing \$ & Nbr_Veh & $\mathrm{CPU}$ \\
\hline GJ1X & 2074 & 674 & 14 & 0,2 & - & 541 & - & 0,08 & 1206 & 406 & 8 & 0,36 \\
\hline GJ2X & 1196 & 596 & 6 & 2,3 & - & 492 & - & 0,08 & 531 & 131 & 4 & 0,36 \\
\hline GJ3X & 2034 & 734 & 13 & 1,5 & - & 638 & - & 0,26 & 1254 & 454 & 8 & 0,38 \\
\hline GJ4X & 2993 & 1193 & 18 & 1,6 & - & 932 & - & 0,61 & 2054 & 854 & 12 & 0,41 \\
\hline GJ5X & 1909 & 909 & 10 & 26,5 & - & 751 & - & 0,62 & 1315 & 715 & 6 & 0,42 \\
\hline GJ6X & 2854 & 954 & 19 & 0,7 & - & 886 & - & 0,6 & 2398 & 1098 & 13 & 0,39 \\
\hline GJ7X & 2573 & 973 & 16 & 1,5 & - & 878 & - & 0,6 & 2092 & 892 & 12 & 0,43 \\
\hline GJ8X & 8326 & 5326 & 30 & 52,2 & - & 3751 & - & 9,56 & 5406 & 3506 & 19 & 2,76 \\
\hline GJ9X & 7026 & 4426 & 26 & 150 & - & 3398 & - & 9,47 & 5332 & 3332 & 20 & 2,79 \\
\hline GJ10X & 7546 & 4446 & 31 & 157 & - & 3311 & - & 6,5 & 4778 & 2878 & 19 & 2,77 \\
\hline GJ11X & 7423 & 4323 & 31 & 40,5 & - & 3263 & - & 9,42 & 4221 & 2321 & 19 & 2,85 \\
\hline \multirow[t]{2}{*}{ Average } & 4178 & 2232 & 19,5 & 39,5 & & 1713 & & 3,4 & 2781 & 1508 & 12,7 & 1,27 \\
\hline & & & & & & & & & $(33.4 \%)^{\mathbf{a}}$ & $(12.0 \%)^{\mathbf{b}}$ & $(34.9 \%)^{\mathbf{c}}$ & \\
\hline GJ1Y & 1814 & 614 & 12 & 0,2 & - & 541 & - & 0,08 & 1052 & 352 & 7 & 0,37 \\
\hline GJ2Y & 1019 & 519 & 5 & 0,3 & - & 492 & - & 0,08 & 539 & 139 & 4 & 0,34 \\
\hline GJ3Y & 2137 & 737 & 14 & 1,4 & - & 638 & - & 0,26 & 1219 & 419 & 8 & 0,45 \\
\hline GJ4Y & 2962 & 1162 & 18 & 1,7 & - & 932 & - & 0,63 & 2032 & 832 & 12 & 0,38 \\
\hline GJ5Y & 1712 & 912 & 8 & 26,5 & - & 751 & - & 0,36 & 1338 & 738 & 6 & 0,42 \\
\hline GJ6Y & 2603 & 1003 & 16 & 3,1 & - & 886 & - & 0,61 & 2197 & 997 & 12 & 0,38 \\
\hline GJ7Y & 2573 & 973 & 16 & 1,5 & - & 878 & - & 0,61 & 2025 & 825 & 12 & 0,45 \\
\hline GJ8Y & 5504 & 4804 & 7 & 24,7 & - & 3751 & - & 9,6 & 5417 & 3517 & 19 & 2,82 \\
\hline GJ9Y & 7601 & 4501 & 31 & 27,8 & - & 3398 & - & 6,54 & 5395 & 3395 & 20 & 2,95 \\
\hline GJ10Y & 7083 & 4183 & 29 & 35,9 & - & 3311 & - & 9,6 & 4883 & 3983 & 19 & 2,92 \\
\hline GJ11Y & 7457 & 4357 & 31 & 40,5 & - & 3263 & - & 6,57 & 4377 & 2477 & 19 & 3,59 \\
\hline \multirow[t]{2}{*}{ Average } & 3860 & 2160 & 17,0 & 14,9 & & 1713 & & 3,2 & 2770 & 1516 & 12,5 & 1,37 \\
\hline & & & & & & & & & $(28.2 \%)^{\mathbf{a}}$ & $(11,5 \%)^{\mathbf{b}}$ & $(26.5 \%)^{\mathbf{c}}$ & \\
\hline \multicolumn{13}{|c|}{${ }^{a}$ The total transportation cost obtained from Salhi and Nagy (1999) improved by GA2 } \\
\hline \multicolumn{13}{|c|}{\begin{tabular}{|l}
${ }^{b}$ The routing cost obtained from Gajpal and Abad (2009) improved by GA2. \\
\end{tabular}} \\
\hline
\end{tabular}

Table 7 reports the results obtained by existing heuristics and GA2 for MD-VRPSDP. In the previous results, those of Gajpal and Abad (2009) are better.

The results show that the performance of the algorithm developed in this paper is better than the performance of previous algorithms. For the instances $\mathrm{X}$, Table 7 shows that our proposed algorithm improves the average value of Gajpal and Abad (2009) by $12 \%$ and for the instances Y, the improvement is $11.5 \%$. And the results, of the number of required vehicles, obtained by GA2 further improve the average values of Salhi and Nagy (1999) by $34.9 \%$ and $26.5 \%$ for instances $\mathrm{X}$ and $\mathrm{Y}$, respectively. It should be noted in particular that the CPU time is considerably much less compared to existing heuristics; an improvement of more than $85 \%$ is observed. Considering these results and CPU times, it can be stated that, the proposed hybrid GA perform well and find good solutions very efficiently. Finding adequate (good enough) solutions in a short time frame is the ultimate goal of GAs, even when the problem size is growing.

\section{CONCLUSION}

MD-VRPSDP-IR is important and practical given the need for integrating forward and reverse flows of material. It is an extension of the VRPSDP which is not yet addressed in the literature. It is a more complicated problem, considering that it needs to tackle multiple depots, inventory restrictions and the VRPSDP problem simultaneously. The considered objective is to minimize the total transportation cost due to the weighted sum of the total distance traveled and the cost related to the number of required vehicles, as mentioned in Section 3 after introducing MD-VRPSPD-IR and its mathematical formulation.

This study contributes to the VRPSDP field by providing an efficient hybrid GA that provides good solutions in a short time frame for MD-VRPSDP-IR. Our contribution in this paper is that we developed a new method, the K-ND heuristic, to assign customers to depots, and we compare its performances with those obtained by the random assignment as well as by the assigning customers to the nearest depot. The proposed algorithm embeds, for each depot as a sub-problem, the Sweep algorithm to construct routes and the Farther Insertion heuristic to improve the solution. Details of the integrity of the proposed method were given in Section 4.

The efficiency of our newly developed heuristic is attested by performance evaluation of the proposed algorithm with computational experiments for MD-VRPSDP-IR and MDVRPSDP. Moreover, according to the results obtained by 
CPLEX, for a small instance, it can be concluded that the proposed Hybrid GA both performs well and is efficient, and gives good and feasible solutions.

Further studies may explore more procedures for assigning customers to depots such as assignment through urgencies which assigns the customers with highest urgency first, that is a way to define a precedence relationship between customers. This work has also to continue testing and comparing other construction and improvement heuristics such as Petal method. Other topics for future work are to include a new crossover and mutation operators, with flexible rates, that will fit more with the nature of the studied problem. Additionally, the proposed method may be applied to a real world routing problems with simultaneous pick-up and deliveries with inventory restrictions.

\section{REFERENCES}

[1] J. Dethloff, "Vehicle routing and reverse logistics: The vehicle routing problem with simultaneous delivery and pick-up", Operations Research Specktrum, vol. 23, pp 79-96, 2001.

[2] J. F. Chen, and T. H. Wu, "Vehicle routing problem with simultaneous deliveries and pickups", Journal of the Operational Research Society, vol. 57, pp 579-587, December 2017.

[3] E. Berhan, "Stochastic vehicle routing problems with simultaneous pickup and delivery services", Journal of Emerging Trends in Computing and Information Sciences, ISSN 2079-8407, vol. 6(7), July 2015.

[4] H. Min, "The MULTIPLE VEHICle ROUTING PROBLEM With SIMULTANEOUS DELIVERY AND PICK-UP POINTS", TRANSPORTATION RESEARCH, VOL. 23(5), PP. 377-386, SEPTEMBER 1989.

[5] R.P. Hornstra, K.J. Roodbergen, and L.C. Coelho, "The Vehicle routing problem with simultaneous pickup and delivery and handling cost", Interuniversity Research Center on Entreprise Networks, Logistics and and Transportation, Working paper $\mathrm{N}^{\circ} 27$, June 2018.

[6] H. Koch, A. Bortfeldt, and G. Wascher, "A hybrid solution approach for the 3L-VRP with simultaneous delivery and pickups", Working paper $\mathrm{N}^{\circ}$ 5, 2017.

[7] Y. Nilufa, "Development of a fuel consumption optimization model for the vehicle routing problem with simultaneous delivery and pickup", Thesis, November 2017.

[8] S. Majidi, S.M. Hosseini-Motlagh, S. Yaghoubi, and A. Jokar, "Fuzzy green vehicle routing problem with simultaneous pickup-delivery and time windows", RAIRO Operations Research, January 2017.

[9] S. Suprayogi, and Y. Priyandari, "Tabu search for the vehicle routing problem with multiple trips, time windows and simultaneous deliverypickup", Jurnal Teknik Industri, vol. 19(2), ISSN 1411-2485, December 2017.

[10] O. Polat, C. B. Kalayci, O. Kulak, and H. O. Günther, "A perturbation based variable neighborhood search heuristic for solving the Vehicle Routing Problem with Simultaneous Pickup and Delivery with Time Limit", European Journal of Operational Research, vol. 242(2), pp. 369382, April 2015.

[11] L. Zhu, and J.B. Sheu, "A parallel simulated annealing method for the vehicle routing problem with simultaneous pickup-delivery and time windows", Computers \& Industrial Engineering, vol. 83, pp.111-122, May 2015.

[12] H. Karimi, "The capacitated hub covering location-routing problem for simultaneous pickup and delivery systems", Computers \& Industrial Engineering, vol. 116, pp. 47-58, February 2018.

[13] C. Lagos, . GGuerrero, E. Cabrera, M.P. Andres, F. Johnson, and F. Paredes, "An improved particle swarm optimization algorithm for the VRP with simultaneous pickup and delivery and time windows", IEEE Latin America Transactions, vol. 16(6), pp.1732-1740, June 2018
[14] H ang, G. Zhijing, Y. Peng, and S. Junqing, "Vehicle routing problem with simultaneous pickups and deliveries and time windows considering fuel consumption and carbon emissions", Chinese Control and Decision Conference, May 2016.

[15] W. Jiahai, Z. Ying, W. Yong, Z. Jun, C.L.P. Chen, and Z. Zibin, "Multiobjective vehicle routing problems with simultaneous delivery and pickup and time windows: formulation, instances and algorithms", IEEE Transactions on Cybernetics, vol. 46(3), pp. 582-594, March 2016.

[16] S. Mostafayi, S. Moazeni, M. Dahmardeh, and K. Mokhtari, "Vehicle routing problem with regard to simultaneous pickup and delivery, time windows and workers assignment on the basis of their abilities and availability", MAGNT Research Report, vol. 3(1), pp. 423-434, January 2015.

[17] AM. Shahdaei, and AM. Rahimi, "Solving vehicule routing problem with simultaneous pick-up and delivery with the application of genetic algirithm", Indian Journal of Fundamental and Applied Life Sciences, vol. 6(S1), pp. 247-259, 2016.

[18] C.X.C.A. Bárbara, P.H. Siqueira, F.A. Giancarlo, and S. Luzia Vidal, "Particle swarm optimization for vehicle routing problem with fleet heterogeneous and simultaneous collection and delivery", Applied Mathematical Sciences, vol. 8(77), pp. 3833 - 3849, 2014.

[19] R. Liu, X. Xie, V. Augusto, and C. Rodriguez, "Heuristic algorithms for a vehicle routing problem with simultaneous delivery and pickup and time windows in home health care", European Journal of Operational Research, Elsevier, vol. 230(3), pp. 475-486, January 2013.

[20] S. Cetin, and C. Gencer, "A Heuristic algorithm for vehicle routing problems with simultaneous pick-up and delivery and hard time windows", Open Journal of Social Sciences, vol.3, pp. 35-41, 2015.

[21] S. Salhi, G. and Nagy, "A cluster insertion heuristic for single and multiple depot vehicle routing problems with backhauling", Journal of the Operational Research Society, vol. 50(10), pp. 1034-1042, September 1999.

[22] G. Nagy, and S. Salhi, "Heuristic algorithms for single and multiple depot vehicle routing problem with pickups and deliveries", European Journal of Operational Research, vol. 162(1), pp.126-141, April 2005.

[23] Y. Gajpal, and P.L. Abad, "Saving based for algorithm for multi-depot version of vehicle routing problem with simultaneous pickup and delivery", International Journal of Enterprize Network Management, vol. 3(3), pp. 201-222, 2009.

[24] J. Li, P.M. Pardalos, H. Sun, J. Peiand, and Y. Zhang, "Iterated local search embedded adaptive neighborhood selection approach for the multi-depot vehicle routing problem with simultaneous deliveries and pickups", Expert Systems with Applications, vol. 42, pp 3551-3561, May 2015.

[25] Y.G. Cai, Y.L. Tang, and Q.J. Yang, “An improved genetic algorithm for multi-depot heterogeneous vehicle routing problem with simultaneous pickup and delivery time windows", Applied Mechanics and Materials, vols. 738-739, pp. 361-365, 2015.

[26] Y. Shimizu, T. Sakaguchi, and J.K. Yoo, "A hybrid method for solving multi-depot VRP with simultaneous pickup and delivery incorporated with weber basis saving heuristic", Journal of Advanced Mechanical Design, Systems, and Manufacturing, vol.10(1), 2016.

[27] S. Karakatic, and V. Podgorelec, "A survey of genetic algorithms for solving multi depot vehicle routing problem", Applied Soft Computing, vol. 27(0), pp. 519-532, February 2015.

[28] B.E. Gillett, and J.G. Johnson, "Multi-terminal vehicle-dispatch algorithm", OMEGA, the International Journal of Management Science, vol. 4(6), pp. 711-718, 1976.

[29] N. Christofides, and S. Eilon, "An algorithm for the vehicle dispatching problems", Operations Research Quarterly, vol. 20, pp. 309-318, September 1969.

[30] B.E. Gillett, and L.R. Miller, "A heuristic algorithm for the vehicledispatch problem", Operations Research, vol. 22(2), pp. 340-349, April 1974. 